\title{
Asymptotics of polygons in restricted geometries subject to a force
}

\author{
Nicholas R. Beaton, Jeremy Eng, and Christine E. Soteros \\ Department of Mathematics and Statistics, University of Saskatchewan, Saskatoon, Canada
}

\begin{abstract}
We consider self-avoiding polygons in a restricted geometry, namely an infinite $L \times M$ tube in $\mathbb{Z}^{3}$. These polygons are subjected to a force $f$, parallel to the infinite axis of the tube. When $f>0$ the force stretches the polygons, while when $f<0$ the force is compressive. In this extended abstract we obtain and prove the asymptotic form of the free energy in the limit $f \rightarrow-\infty$. We conjecture that the $f \rightarrow-\infty$ asymptote is the same as the free energy of Hamiltonian polygons, which visit every vertex in a $L \times M \times N$ box.
\end{abstract}

Résumé. Nous considérons des polygones auto-évitants dans une géométrie restreinte, en particulier un tube infini dans $\mathbb{Z}^{3}$ avec les dimensions $L \times M$. Ces polygones sont soumis à une force $f$, parallèle à l'axe infini du tube. Quand $f>0$ la force tend les polygones, alors que quand $f<0$ la force est compressive. Dans ce résumé détaillé, nous obtenons et prouvons la forme asymptotique de l'énergie libre en la limite $f \rightarrow-\infty$. Nous conjecturons que l'asymptote $f \rightarrow-\infty$ est la même que l'énergie libre des polygones hamiltoniennes, qui visitent chaque sommet dans un boîte $L \times M \times N$.

Keywords. statistical mechanics, polygons, polymers

\section{Introduction}

Self-avoiding walks and polygons are the standard lattice models of, respectively, linear and ring polymers in dilute solution (Vanderzande (1998)). While there have been many important recent breakthroughs in the study of these models for dimensions $d \geq 4$ and $d=2$ (see e.g. Bauerschmidt et al. (2012)) many challenging questions remain open (especially for $d=3$ ) with regard to phase transitions and entanglement complexity (Orlandini and Whittington (2007)).

Regarding entanglement complexity, using a self-avoiding polygon model, Sumners and Whittington (1988) and independently Pippenger (1989) proved the 1960's Frisch-Wasserman-Delbruck (FWD) conjecture that sufficiently long ring polymers will be knotted (see e.g. Sumners and Whittington (1988)). Since then, motivated in part by experimental studies of DNA, there has been much interest in exploring entanglement complexity in polymer models and the effect of factors such as solvent quality, external forces and/or geometric confinement (Orlandini and Whittington (2007)). In particular, motivated by atomic force microscopy experiments, one such area of interest has been models of polymers subject to a tensile force $f$ (see e.g. Farago et al. (2002); Krawczyk et al. (2005); Janse van Rensburg et al. (2008); Atapour et al. (2009); Ioffe and Velenik (2010); Beaton (2015); Beaton et al. (2015)). For this case, 
Janse van Rensburg et al. (2008) found that for sufficiently large fixed forces and sufficiently large polygons, all but exponentially few are knotted, i.e. the FWD-conjecture holds for sufficiently large forces. It is believed that this should hold for any force, however this has yet to be proved. By restricting the polygons to lie in a lattice tube, however, Atapour et al. (2009) proved that for any fixed force (either stretching $(f>0)$ or compressing $(f<0))$, all but exponentially few sufficiently large polygons are knotted (i.e. the FWD-conjecture holds). The proof was based on transfer-matrix theory and pattern theorem arguments.

Atapour et al. (2009) considered self-avoiding polygons in a restricted geometry, namely an infinite $L \times M$ tube in $\mathbb{Z}^{3}$, which were subjected to a force $f$ parallel to the infinite axis of the tube. One question left open in that study was the behaviour of the polygons as $f \rightarrow-\infty$. In this case, for the FWDconjecture, for example, there are two limits involved, the limit as the polygon length $n \rightarrow \infty$ and the limit as the force $f \rightarrow-\infty$. If one fixes a polygon's length $n$, and lets the force go to $-\infty$, then the result is a polygon of length $n$ which is maximally compressed and hence its extent in the infinite axis of the tube is $\lceil n /(L+1)(M+1)\rceil$. If $n$ (even) is a multiple of $(L+1)(M+1)$, the resulting polygon corresponds to an undirected Hamiltonian circuit (which we call a Hamiltonian polygon) on a rectangular sub-lattice of the tube. Eng (2014) proved that the FWD-conjecture holds for Hamiltonian polygons. However, it is unclear what happens if one instead first takes $n \rightarrow \infty$ and then increases the compressive force. That is, the behaviour of the limiting free energy in the $f \rightarrow-\infty$ limit has been an open question, even for this simpler, essentially one-dimensional, problem.

In this extended abstract, we explore the Atapour et al. (2009) model further to address this question by investigating the behaviour of the model's free energy as $f \rightarrow-\infty$. In particular, we establish the existence and form of the asymptote in this limit. In Section 2 we precisely define the model and state some existing results regarding the thermodynamic limit. In Section 3 we state and prove the main theorem (Theorem 11). Our proof depends heavily on some results by Janse van Rensburg (2000) regarding the density functions of lattice models; for readability we quote them here.

This paper is part of a larger work Beaton et al. (2016), in which we also determine the asymptotic form of the free energy as $f \rightarrow \infty$, as well as conduct a more detailed investigation of Hamiltonian polygons. We conjecture that the asymptote found in this paper is in fact the free energy of these Hamiltonian polygons and hence that the FWD conjecture will also hold in the $f \rightarrow-\infty$ limit. A brief discussion of this conjecture can be found in Section 4 Note that although the presentation here is focused on polygons in tubular sublattices of $\mathbb{Z}^{3}$, the proofs presented include the case that one of $L$ or $M$ is zero so that the tube is actually a slit in $\mathbb{Z}^{2}$. Similar arguments are expected to hold for polygons in tubular sublattices of $\mathbb{Z}^{d}$, for any $d \geq 4$, however it is only for $d=3$ that polygons can be knotted.

\section{The model}

Let $\mathbb{T}_{L, M} \equiv \mathbb{T} \subset \mathbb{Z}^{3}$ be the semi-infinite $L \times M$ tube on the cubic lattice defined by

$$
\mathbb{T}=\left\{(x, y, z) \in \mathbb{Z}^{3}: x \geq 0,0 \leq y \leq L, 0 \leq z \leq M\right\} .
$$

We will assume without loss of generality that $L \geq M$. Define $\mathcal{P}_{\mathbb{T}}$ to be the set of self-avoiding polygons in $\mathbb{T}$ which occupy at least one vertex in the plane $x=0$, and let $\mathcal{P}_{\mathbb{T}, n}$ be the subset of $\mathcal{P}_{\mathbb{T}}$ comprising polygons with $n$ edges. Then let $p_{\mathbb{T}, n}=\left|\mathcal{P}_{\mathbb{T}, n}\right|$. See Figure 1 for an example.

Remark. Throughout the rest of this paper, the symbol $n$ will only be used to denote the number of edges in polygons. We will thus always assume that $n$ is even. This includes limits, where for example $\lim _{n \rightarrow \infty}$ should be interpreted as a limit through even values of $n$ only. 


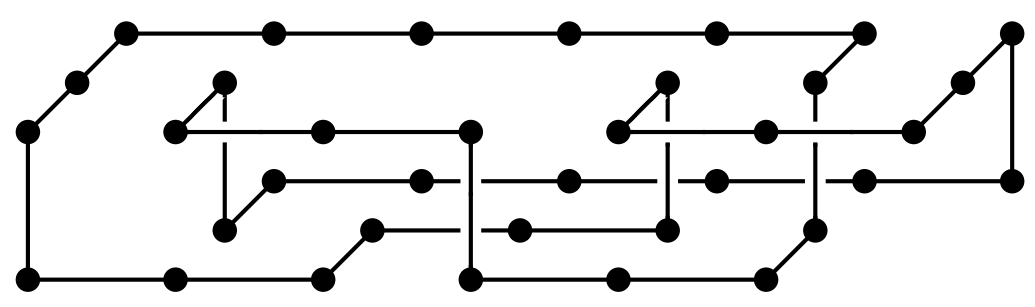

Fig. 1: A self-avoiding polygon in the $2 \times 1$ tube. This polygon has length 36 and span 6 .

It is known (e.g. Atapour et al. (2009) ) that the connective constant of polygons in $\mathbb{T}$,

$$
\kappa_{\mathbb{T}}=\lim _{n \rightarrow \infty} \frac{1}{n} \log p_{\mathbb{T}, n},
$$

exists and is finite.

We define the span $s(\pi)$ of a polygon $\pi \in \mathcal{P}_{\mathbb{T}}$ to be the maximal $x$-coordinate reached by any of its vertices. To model a force acting parallel to the $x$-axis, we associate a Boltzmann weight $e^{f s(\pi)}$ with each polygon $\pi$. Let $p_{\mathbb{T}, n}(s)$ be the number of polygons in $\mathcal{P}_{\mathbb{T}, n}$ of span $s$. We define the partition function

$$
Z_{\mathbb{T}, n}(f)=\sum_{|\pi|=n} e^{f s(\pi)}=\sum_{s} p_{\mathbb{T}, n}(s) e^{f s} .
$$

The weight $f$ represents a force as follows: when $f \ll 0$, polygons with small span will dominate the partition function, so this corresponds to the "compressed" regime. On the other hand, when $f \gg 0$, polygons with large span will dominate the partition function, corresponding to the "stretched" regime.

The free energy of polygons in $\mathbb{T}$ is defined as

$$
\mathcal{F}_{\mathbb{T}}(f)=\lim _{n \rightarrow \infty} \frac{1}{n} \log Z_{\mathbb{T}, n}(f) .
$$

Atapour et al. (2009) showed that $\mathcal{F}_{\mathbb{T}}(f)$ exists for all $f$. It is a convex function of $f$, and is thus continuous and almost-everywhere differentiable.

Note that for this model, the grand canonical partition function is given by

$$
G_{\mathbb{T}}(f, z)=\sum_{n} Z_{\mathbb{T}, n}(f) e^{z n}=\sum_{n} \sum_{s} p_{\mathbb{T}, n}(s) e^{f s} e^{z n},
$$

which is a two-variable generating function for $p_{\mathbb{T}, n}(s)$. For fixed $f$, the free energy defined above determines the radius of convergence of $G_{\mathbb{T}}(f, z)$; specifically, $G_{\mathbb{T}}(f, z)$ converges for all $z<-\mathcal{F}_{\mathbb{T}}(f)$. Transfer-matrix arguments given in Atapour (2008); Atapour et al. (2009) establish that $G_{\mathbb{T}}(f, z)$ is a rational function in $e^{f}$ and $e^{z}$.

\section{$3 f \rightarrow-\infty$ asymptote}

In this section we consider the case of compressed polygons. That is, we investigate the behaviour of the free energy $\mathcal{F}_{\mathbb{T}}(f)$ in the limit $f \rightarrow-\infty$. Some preliminary definitions and results are required before the main theorem can be stated. 


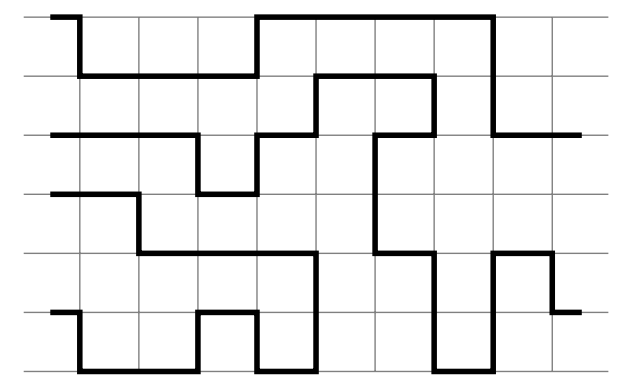

Fig. 2: A 9-block of the $6 \times 0$ tube. This 9-block has length 50 .

Given a polygon $\pi \in \mathbb{T}$, a hinge $H_{k}$ of $\pi$ is the set of edges and vertices lying in the intersection of $\pi$ and the $y$ - $z$ plane defined by $\{(x, y, z): x=k\}$. A section $S_{k}$ is the set of edges in $\pi$, in the $x$ direction, connecting $H_{k-1}$ and $H_{k}$. A half-section of $S_{k}$ is the set of half-edges in $S_{k}$ with either $k-1 \leq x \leq k-\frac{1}{2}$ or $k-\frac{1}{2} \leq x \leq k$.

A 1-block of $\mathbb{T}$ is any non-empty hinge which can occur in a polygon $\pi$ in $\mathbb{T}$, together with any halfedges of $\pi$ in the two adjacent half-sections. The length of a 1-block is the sum of the lengths of all its edges and half-edges. It is thus natural to view a 1-block as the part of a polygon between two half-integer planes with $x$-coordinates $k \pm \frac{1}{2}$ with $k \in \mathbb{Z}$.

An $s$-block is then any connected sequence of $s$-blocks, the entirety of which can occur in a polygon in $\mathbb{T}$. The length of an $s$-block is the sum of the lengths of its constituent 1-blocks. Let $b_{\mathbb{T}, s}$ be the number of $s$-blocks in $\mathbb{T}$, counted up to translation in the $x$-direction. See Figure 2 for an example.

Lemma 1 The limit

$$
\beta_{\mathbb{T}}=\lim _{s \rightarrow \infty} \frac{1}{s} \log b_{\mathbb{T}, s}
$$

exists and is finite.

Proof: Any $(s+t)$-block can be cut into an $s$-block and a $t$-block; we thus have

$$
b_{\mathbb{T}, s+t} \leq b_{\mathbb{T}, s} b_{\mathbb{T}, t} .
$$

So $\left\{\log b_{\mathbb{T}, s}\right\}$ is a subadditive sequence, and the limit (3) exists. We clearly have $b_{\mathbb{T}, s} \geq 1$ for all $s \geq 1$, so that $\beta_{\mathbb{T}}$ is finite.

A 1-block is called full (no empty vertices) if its length is equal to $W=(L+1)(M+1)$. An $s$-block is full if every one of its constituent 1-blocks is full. Let $b_{\mathbb{T}, s}^{0}$ be the number of full $s$-blocks in $\mathbb{T}$. The following lemma can be proved in the same way as Lemma 1 .

Lemma 2 The limit

$$
\beta_{\mathbb{T}}^{0}=\lim _{s \rightarrow \infty} \frac{1}{s} \log b_{\mathbb{T}, s}^{0}
$$

exists and is finite.

We are now able to state the main theorem of this section. 
Theorem 1 For any tube size $L \times M$, in the limit $f \rightarrow-\infty$ the free energy $\mathcal{F}_{\mathbb{T}}(f)$ is asymptotic to $\left(\beta_{\mathbb{T}}^{0}+f\right) / W$, where $W=(L+1)(M+1)$. That is,

$$
\lim _{f \rightarrow-\infty}\left(\mathcal{F}_{\mathbb{T}}(f)-\frac{f}{W}\right)=\frac{\beta_{\mathbb{T}}^{0}}{W} .
$$

\subsection{Density functions}

There are a number of results from Chapter 3 of Janse van Rensburg (2000) which will be important for the proof of Theorem 1. For this reason we explicitly state them here. We begin with some necessary assumptions. For the remainder of this section $k$ and $m$ will be non-negative integers representing respectively the size and energy of an object. For the models in this paper, an example object is a polygon, with its size being its length $n$ and its energy being its span $s$; however, we will also consider the opposite scenario where a polygon's size is given by its span and its energy by its length.

Assumptions 1 (Assumptions 3.1 of Janse van Rensburg (2000)) Let $u_{k}(m)$ be the number of objects of size $k$ and energy $m$. Assume that $u_{k}(m)$ satisfies the following properties:

(1) There exists a constant $K>0$ such that $0 \leq u_{k}(m) \leq K^{k}$ for each value of $k$ and $m$.

(2) There exist finite integers $A_{k}$ and $B_{k}$ and a real constant $C$ satisfying $0 \leq A_{k} \leq B_{k} \leq C k$ such that $u_{k}(m)>0$ for $A_{k} \leq m \leq B_{k}$ and $u_{k}(m)=0$ otherwise.

(3) The values $u_{k}(m)$ satisfy the following supermultiplicative inequality for each value of $k_{1}, m_{1}, k_{2}, m_{2}$ such that $u_{k_{1}}\left(m_{1}\right)>0$ and $u_{k_{2}}\left(m_{2}\right)>0$ :

$$
u_{k_{1}}\left(m_{1}\right) u_{k_{2}}\left(m_{2}\right) \leq u_{k_{1}+k_{2}}\left(m_{1}+m_{2}\right)
$$

We now add a further assumption which is not required in Janse van Rensburg (2000), but will make calculations here somewhat simpler.

Assumptions 2 The following limits exist, and satisfy

$$
A=\lim _{k \rightarrow \infty} \frac{A_{k}}{k}<\lim _{k \rightarrow \infty} \frac{B_{k}}{k}=B
$$

Theorem 2 (Theorems 3.4 and 3.5 of Janse van Rensburg (2000)) Let $u_{k}(m)$ be a sequence satisfying Assumptions 1 and 2. Then if $\epsilon \in(A, B)$, the density function $\mathcal{D}(\epsilon)$ is defined by the limit

$$
\log \mathcal{D}(\epsilon)=\lim _{k \rightarrow \infty} \frac{1}{k} \log u_{k}(\lfloor\epsilon k\rfloor)
$$

The function $\log \mathcal{D}(\epsilon)$ is concave in $\epsilon$ over $(A, B)$, and is continuous and almost-everywhere differentiable.

We next define partition functions and relate them to the density function $\mathcal{D}(\epsilon)$. Let

$$
U_{k}(z)=\sum_{m} u_{k}(m) e^{z m}
$$


Theorem 3 (Theorems 3.6, 3.17 and 3.19 of Janse van Rensburg(2000)) The limit

$$
\mathcal{E}(z)=\lim _{n \rightarrow \infty} \frac{1}{k} \log U_{k}(z)
$$

exists for all z. Moreover,

$$
\mathcal{E}(z)=\sup _{A<\epsilon<B}\{\log \mathcal{D}(\epsilon)+\epsilon z\} .
$$

Our final preliminary result is a generalisation of equation (3.4) in Janse van Rensburg (2000). For brevity the proof is omitted in this extended abstract, but will be published with the full version of the paper elsewhere.

Lemma 3 Let $T_{k}$ be a sequence satisfying $A_{k} \leq T_{k} \leq B_{k}$ and $T_{k}=B k+o(k)$. Moreover, assume that $B_{k}<B k$ for all $k$ sufficiently large. Then

$$
\log \mathcal{D}\left(B^{-}\right):=\lim _{\epsilon \rightarrow B^{-}} \log \mathcal{D}(\epsilon) \geq \limsup _{k \rightarrow \infty} \frac{1}{k} \log u_{k}\left(T_{k}\right) .
$$

We also note the following consequence (see for example (Janse van Rensburg, 2000, equation (3.15))) of the concavity of $\log \mathcal{D}(\epsilon)$ and Theorem 3 .

$$
\mathcal{E}(z) \sim \lim _{\epsilon \rightarrow B^{-}} \log \mathcal{D}(\epsilon)+\epsilon z \quad \text { as } \quad z \rightarrow \infty, \quad \text { and } \quad \mathcal{E}(z) \sim \lim _{\epsilon \rightarrow A^{+}} \log \mathcal{D}(\epsilon)+\epsilon z \quad \text { as } \quad z \rightarrow-\infty
$$

\subsection{The proof}

We begin with some more definitions. Let $\mathcal{P}_{\mathbb{T}}^{*}$ be the set of those polygons $\pi \in \mathcal{P}_{\mathbb{T}}$ which satisfy the additional constraints:

- $\pi$ has $\operatorname{span} s \geq 2$,

- $\pi$ contains the edge $(0,0,0)-(0,1,0)$ and no other edges in the plane $x=0$,

- $\pi$ contains the edge $(s, 0,0)-(s, 1,0)$ and no other edges in the plane $x=s$, and

- $\pi$ contains no edges in the plane $x=s-1$.

Let $p_{\mathbb{T}, n}^{*}(s)$ be the number of polygons in $\mathcal{P}_{\mathbb{T}}^{*}$ with length $n$ and span $s$. We define a partition function analogous to $Z_{\mathbb{T}, n}(f)$ :

$$
Z_{\mathbb{T}, n}^{*}(f)=\sum_{s} p_{\mathbb{T}, n}^{*}(s) e^{f s} .
$$

Lemma $4 \mathcal{P}_{\mathbb{T}}^{*}$ polygons have a free energy

$$
\mathcal{F}_{\mathbb{T}}^{*}(f)=\lim _{n \rightarrow \infty} \frac{1}{n} \log Z_{\mathbb{T}, n}^{*}(f) .
$$

Moreover, $\mathcal{F}_{\mathbb{T}}^{*}(f)=\mathcal{F}_{\mathbb{T}}(f)$. 
Proof: If $(L, M)=(1,0)$ then $Z_{\mathbb{T}, n}^{*}(f)=e^{f(n-2) / 2}$, and the result is trivial. Otherwise, at least one of the statements $L \geq 2$ or $M \geq 1$ is true, and hence also $W>2$.

We show that the sequence $p_{\mathbb{T}, n}^{*}(s)$ satisfies Assumptions 1 and 2 with size $k=n$ and energy $m=s$, so that Theorem 3 can be applied.

(1) Using $K=6$ suffices to satisfy condition (1).

(2) The numbers $A_{n}$ and $B_{n}$ (respectively the minimum and maximum possible spans for a $\mathcal{P}_{\mathbb{T}}^{*}$ polygon of length $n$ ) are

$$
A_{n}=\left\lceil\frac{n-6}{W}\right\rceil+2 \quad B_{n}=\frac{n-2}{2} .
$$

It can be shown by construction that $p_{\mathbb{T}, n}^{*}(s)>0$ for each even $n$ and each $s$ such that $A_{n} \leq$ $s \leq B_{n}$ so that taking $C=1 / 2$ gives condition (2). Note that $A=\lim _{n \rightarrow \infty} A_{n} / n=1 / W$ and $B=\lim _{n \rightarrow \infty} B_{n} / n=1 / 2$ with $A<B$ for any $W>2$, so that Assumptions 2 also hold.

(3) The set $\mathcal{P}_{\mathbb{T}}^{*}$ has been defined so that any two polygons $\pi_{1}, \pi_{2}$ in $\mathcal{P}_{\mathbb{T}}^{*}$ can be concatenated in a way that preserves both total length and total span. Let $\pi_{1}$ have span $s_{1}$, and define $e_{1}$ to be the single edge of $\pi_{1}$ with maximal $x$-coordinate and $e_{2}$ to be the edge of $\pi_{2}$ with minimal $x$-coordinate. Then

i. Translate $\pi_{2}$ so that $e_{1}$ and $e_{2}$ coincide, and delete those two edges.

ii. If $L \geq 2$ then replace the edge $\left(s_{1}-1,1,0\right)-\left(s_{1}, 1,0\right)$ with the three edges

$$
\left(s_{1}-1,1,0\right)-\left(s_{1}-1,2,0\right)-\left(s_{1}, 2,0\right)-\left(s_{1}, 1,0\right) .
$$

Otherwise if $(L, M)=(1,1)$ then replace the edge $\left(s_{1}-1,1,0\right)-\left(s_{1}, 1,0\right)$ with the edges

$$
\left(s_{1}-1,1,0\right)-\left(s_{1}-1,1,1\right)-\left(s_{1}, 1,1\right)-\left(s_{1}, 1,0\right) .
$$

So any two polygons $\pi_{1}, \pi_{2}$ in $\mathcal{P}_{\mathbb{T}}^{*}$, of lengths $n_{1}$ and $n_{2}$ and spans $s_{1}$ and $s_{2}$, can be concatenated to give another polygon in $\mathcal{P}_{\mathbb{T}}^{*}$, of length $n_{1}+n_{2}$ and span $s_{1}+s_{2}$. Thus

$$
p_{\mathbb{T}, n_{1}}^{*}\left(s_{1}\right) p_{\mathbb{T}, n_{2}}^{*}\left(s_{2}\right) \leq p_{\mathbb{T}, n_{1}+n_{2}}^{*}\left(s_{1}+s_{2}\right) .
$$

Since $\mathcal{P}_{\mathbb{T}}^{*} \subseteq \mathcal{P}_{\mathbb{T}}$, we have $\mathcal{F}_{\mathbb{T}}^{*}(f) \leq \mathcal{F}_{\mathbb{T}}(f)$. For brevity we omit the proof of the reverse inequality; it follows from the fact that any $\mathcal{P}_{\mathbb{T}}$ polygon can be converted into a $\mathcal{P}_{\mathbb{T}}^{*}$ polygon by adding a fixed number of edges which extend the span by no more than a constant amount.

Polygons in $\mathcal{P}_{\mathbb{T}}^{*}$ then have a density function

$$
\log \mathcal{S}_{\mathbb{T}}^{*}(\epsilon)=\lim _{n \rightarrow \infty} \frac{1}{n} \log p_{\mathbb{T}, n}^{*}(\lfloor\epsilon n\rfloor)
$$

with

$$
\mathcal{F}_{\mathbb{T}}^{*}(f)=\sup _{1 / W<\epsilon<1 / 2}\left\{\log \mathcal{S}_{\mathbb{T}}^{*}(\epsilon)+\epsilon f\right\}
$$


The approach to proving Theorem 1 will involve the 'dual' object to $\mathcal{F}_{\mathbb{T}}^{*}(f)$. Let $q_{\mathbb{T}, s}^{*}(n)=p_{\mathbb{T}, n}^{*}(s)$. (We introduce this quantity to make it clear that we are now interpreting the span of a polygon as its 'size' and the length of a polygon as its 'energy'.) Define

$$
Q_{\mathbb{T}, s}^{*}(z)=\sum_{n} q_{\mathbb{T}, s}^{*}(n) e^{z n}
$$

Lemma 5 The free energy

$$
\mathcal{G}_{\mathbb{T}}^{*}(z)=\lim _{s \rightarrow \infty} \frac{1}{s} \log Q_{\mathbb{T}, s}^{*}(z)
$$

exists for all $z$.

Proof: The case $(L, M)=(1,0)$ is again trivial, so we can take at least one of $L \geq 2$ or $M \geq 1$ to be true.

We show that the sequence $q_{\mathbb{T}, s}^{*}(n)$ satisfies Assumptions 1 , with one minor caveat.

(1) Since $q_{\mathbb{T}, s}^{*}(n) \leq b_{\mathbb{T}, s+1}$, using $K=\left(b_{\mathbb{T}, 1}\right)^{2}$ suffices to satisfy condition (1).

(2) The numbers $A_{s}$ and $B_{s}$ (respectively the minimum and maximum possible lengths of a $\mathcal{P}_{\mathbb{T}}^{*}$ polygon of span $s$ ) are

$$
A_{s}=2(s+1) \quad B_{s}= \begin{cases}W(s-2)+6 & \text { if } W \text { or } s \text { even } \\ W(s-2)+5 & \text { if } W \text { and } s \text { odd }\end{cases}
$$

and $C=W+6$. However, note that $q_{\mathbb{T}, s}^{*}(n)>0$ only if $n$ is even. Condition (2) can then be met by letting the energy of a polygon be its half-length, rather than its length. Adjusting everything to account for this essentially amounts to taking $n \mapsto n / 2$ in the definitions of $q_{\mathbb{T}, s}^{*}(n)$ and $Q_{\mathbb{T}, s}^{*}(z)$, and likewise dividing the values of $A_{s}$ and $B_{s}$ by 2 . This is straightforward, so we will in general continue to use length instead of half-length. Clearly Assumptions 2 hold with $A=2$ and $B=W$.

(3) The inequality 8 can be rewritten as

$$
q_{\mathbb{T}, s_{1}}^{*}\left(n_{1}\right) q_{\mathbb{T}, s_{2}}^{*}\left(n_{2}\right) \leq q_{\mathbb{T}, s_{1}+s_{2}}^{*}\left(n_{1}+n_{2}\right) .
$$

It is now the asymptotic behaviour of $\mathcal{G}_{\mathbb{T}}^{*}(z)$ as $z \rightarrow \infty$ in which we are interested, and we will see later that this is related, in a very simple way, to the behaviour of $\mathcal{F}_{\mathbb{T}}(f)$ as $f \rightarrow-\infty$. We once again make use of a density function. By Theorem 3 we have a 'length density' function:

$$
\log \mathcal{L}_{\mathbb{T}}^{*}(\alpha)=\lim _{s \rightarrow \infty} \log q_{\mathbb{T}, s}^{*}(\lfloor\alpha s\rfloor) .
$$

The function $\log \mathcal{L}_{\mathbb{T}}^{*}(\alpha)$ is finite and concave for $\alpha \in(2, W)$. The inverse Legendre transform is then

$$
\mathcal{G}_{\mathbb{T}}^{*}(z)=\sup _{2<\alpha<W}\left\{\log \mathcal{L}_{\mathbb{T}}^{*}(\alpha)+\alpha z\right\}
$$

From (7) it follows that the behaviour of $\mathcal{G}_{\mathbb{T}}^{*}(z)$ as $z \rightarrow \infty$ is determined by the behaviour of $\log \mathcal{L}_{\mathbb{T}}^{*}(\alpha)$ as $\alpha \rightarrow W^{-}$. This behaviour is explored next by obtaining upper and lower bounds. 
Lemma 6 For any tube size $L \times M$, the density function $\mathcal{L}_{\mathbb{T}}^{*}(\alpha)$ satisfies

$$
\log \mathcal{L}_{\mathbb{T}}^{*}\left(W^{-}\right):=\lim _{\alpha \rightarrow W^{-}} \log \mathcal{L}_{\mathbb{T}}^{*}(\alpha) \leq \beta_{\mathbb{T}}^{0} .
$$

Proof: The following argument is inspired by a proof of Rychlewski and Whittington (2011) regarding adsorbing self-avoiding walks.

Let $j_{\mathbb{T}, s}^{*}(m)$ be the number of $\mathcal{P}_{\mathbb{T}}^{*}$ polygons of span $s$ and length at least $m$. Write $s+1=p r+q$ with $0 \leq q<r$, and think of a polygon of span $s$ as the connected sequence of $p r$-blocks and (possibly) one $q$-block.

If a polygon has span $s$ and length $n$ then it has $W(s+1)-n$ unoccupied vertices within its $s+1$ hinges. We will consider $j_{\mathbb{T}, s}^{*}(\lfloor\alpha s\rfloor)$, so define $u=W(s+1)-\lfloor\alpha s\rfloor$, the maximum number of unoccupied vertices in a polygon with at least length $\lfloor\alpha s\rfloor$. Considering all possible choices for the number $k$ of $r$-blocks with unoccupied vertices, we have

$$
j_{\mathbb{T}, s}^{*}(\lfloor\alpha s\rfloor) \leq \sum_{k=0}^{u}\left(\begin{array}{l}
p \\
k
\end{array}\right)\left(b_{\mathbb{T}, r}\right)^{k}\left(b_{\mathbb{T}, r}^{0}\right)^{p-k} b_{\mathbb{T}, q} .
$$

For $\delta>0$ take $r$ sufficiently large so that $b_{\mathbb{T}, r} \leq e^{\left(\beta_{\mathbb{T}}+\delta\right) r}$ and $b_{\mathbb{T}, r}^{0} \leq e^{\left(\beta_{\mathbb{T}}^{0}+\delta\right) r}$. Then

$$
\begin{aligned}
j_{\mathbb{T}, s}^{*}(\lfloor\alpha s\rfloor) & \leq b_{\mathbb{T}, q} \sum_{k=0}^{u}\left(\begin{array}{l}
p \\
k
\end{array}\right) e^{k r\left(\beta_{\mathbb{T}}+\delta\right)} e^{(p-k) r\left(\beta_{\mathbb{T}}^{0}+\delta\right)} \\
& =b_{\mathbb{T}, q} e^{r p\left(\beta_{\mathbb{T}}^{0}+\delta\right)} \sum_{k=0}^{u}\left(\begin{array}{l}
p \\
k
\end{array}\right) e^{k r\left(\beta_{\mathbb{T}}-\beta_{\mathbb{T}}^{0}\right)} .
\end{aligned}
$$

Noting that $p \sim s / r$, take $\alpha$ sufficiently close to $W$ so that $u<p / 2$. Then the largest summand of (13) is the last one, so

$$
j_{\mathbb{T}, s}^{*}(\lfloor\alpha s\rfloor) \leq b_{\mathbb{T}, q} e^{r p\left(\beta_{\mathbb{T}}^{0}+\delta\right)}(u+1)\left(\begin{array}{l}
p \\
u
\end{array}\right) e^{r u\left(\beta_{\mathbb{T}}-\beta_{\mathbb{T}}^{0}\right)} .
$$

Take logs, divide by $s$ and apply Stirling's approximation:

$$
\begin{aligned}
\frac{1}{s} \log j_{\mathbb{T}, s}^{*}(\lfloor\alpha\rfloor) \leq \frac{1}{s} \log b_{\mathbb{T}, q}+\frac{r p\left(\beta_{\mathbb{T}}^{0}+\delta\right)}{s} & +\frac{r u\left(\beta_{\mathbb{T}}-\beta_{\mathbb{T}}^{0}\right)}{s}+\frac{1}{s} \log (u+1) \\
& -\frac{p}{s} \log \left(\frac{p-u}{p}\right)+\frac{u}{s} \log \left(\frac{p-u}{u}\right)+O\left(\frac{\log s}{s}\right) .
\end{aligned}
$$

With $r$ fixed, take a limsup as $s \rightarrow \infty$, to get

$$
\begin{aligned}
\log \mathcal{L}_{\mathbb{T}}^{*}(\alpha) & \leq \limsup _{s \rightarrow \infty} \frac{1}{s} \log j_{\mathbb{T}, s}^{*}(\lfloor\alpha s\rfloor) \\
& \leq \beta_{\mathbb{T}}^{0}+\delta+r(W-\alpha)\left(\beta_{\mathbb{T}}-\beta_{\mathbb{T}}^{0}\right)-\frac{1}{r} \log (1-r(W-\alpha))+(W-\alpha) \log \left(\frac{1}{r(W-\alpha)}-1\right) .
\end{aligned}
$$

Now taking $\alpha \rightarrow W^{-}$, we find

$$
\log \mathcal{L}_{\mathbb{T}}^{*}\left(W^{-}\right) \leq \beta_{\mathbb{T}}^{0}+\delta
$$

Since $\delta$ can be arbitrarily small, the proof is complete. 
Lemma 7 For any tube size $L \times M$, the density function $\mathcal{L}_{\mathbb{T}}^{*}(\alpha)$ satisfies

$$
\log \mathcal{L}_{\mathbb{T}}^{*}\left(W^{-}\right)=\lim _{\alpha \rightarrow W^{-}} \log \mathcal{L}_{\mathbb{T}}^{*}(\alpha) \geq \beta_{\mathbb{T}}^{0}
$$

Proof: By definition, any $s$-block or full $s$-block can be 'completed', by adding edges at one or both ends, to form a self-avoiding polygon of span $\geq s+1$. In particular, there exist constants $t$ and $c$ so that any full $s$-block can be completed to a unique $\mathcal{P}_{\mathbb{T}}^{*}$ polygon of span $s+t$ and length between $W s$ and $W s+c$. So

$$
b_{\mathbb{T}, s}^{0} \leq \sum_{n=W s}^{W s+c} q_{\mathbb{T}, s+t}^{*}(n) .
$$

Now let $n_{s+t}^{\max }$ be the value of $n$ between $W s$ and $W s+c$ which maximises $q_{\mathbb{T}, s+t}^{*}(n)$ (if there are multiple such values, take the smallest one). We then have

$$
b_{\mathbb{T}, s}^{0} \leq(c+1) q_{\mathbb{T}, s+t}^{*}\left(n_{s+t}^{\max }\right) .
$$

Observe that $n_{s}^{\max }$ is a sequence which satisfies the conditions of Lemma 3 it is by definition a value between the minimum and maximum lengths for $\mathcal{P}_{\mathbb{T}}^{*}$ polygons of $\operatorname{span} s$, and $n_{s}^{\max }=W s+o(s)$. So

$$
\log \mathcal{L}_{\mathbb{T}}^{*}\left(W^{-}\right) \geq \limsup _{s \rightarrow \infty} \frac{1}{s} \log q_{\mathbb{T}, s}^{*}\left(n_{s}^{\max }\right) \geq \limsup _{s \rightarrow \infty} \frac{1}{s} \log \left(\frac{b_{\mathbb{T}, s-t}^{0}}{c+1}\right)=\lim _{s \rightarrow \infty} \frac{1}{s} \log b_{\mathbb{T}, s}^{0}=\beta_{\mathbb{T}}^{0} .
$$

Corollary 1 In the limit as $z \rightarrow \infty$, the free energy $\mathcal{G}_{\mathbb{T}}^{*}(z)$ is asymptotic to $W z+\beta_{\mathbb{T}}^{0}$. That is,

$$
\lim _{z \rightarrow \infty}\left(\mathcal{G}_{\mathbb{T}}^{*}(z)-W z\right)=\beta_{\mathbb{T}}^{0} .
$$

Proof: By (7), for $z \rightarrow \infty$ in (11), the supremum is found by taking $\alpha \rightarrow W^{-}$. In this case $\log \mathcal{L}_{\mathbb{T}}^{*}(\alpha) \rightarrow$ $\beta_{\mathbb{T}}^{0}$.

It now remains to establish the relationship between the two density functions $\mathcal{S}_{\mathbb{T}}^{*}(\epsilon)$ and $\mathcal{L}_{\mathbb{T}}^{*}(\alpha)$.

Proof of Theorem 1; For given rational $\alpha \in(2, W)$, we have

$$
\log \mathcal{L}_{\mathbb{T}}^{*}(\alpha)=\lim _{s \rightarrow \infty} \frac{1}{s} \log q_{\mathbb{T}, s}^{*}(\lfloor\alpha s\rfloor) .
$$

If we take this limit through values of $s$ such that $s / \alpha$ is an integer, then this can be written as

$$
\log \mathcal{L}_{\mathbb{T}}^{*}(\alpha)=\lim _{s \rightarrow \infty} \frac{1}{s / \alpha} \log q_{\mathbb{T}, s / \alpha}^{*}(s)=\lim _{s \rightarrow \infty} \frac{\alpha}{s} \log p_{\mathbb{T}, s}^{*}(s / \alpha)=\alpha \log \mathcal{S}_{\mathbb{T}}^{*}(1 / \alpha) .
$$

Continuity extends this to all $\alpha \in(2, W)$, and for $\epsilon \in(1 / W, 1 / 2)$ it can alternatively be written as

$$
\epsilon \log \mathcal{L}_{\mathbb{T}}^{*}(1 / \epsilon)=\log \mathcal{S}_{\mathbb{T}}^{*}(\epsilon) .
$$


Now consider (9) in the case that $f \rightarrow-\infty$. From (7), we find the supremum by taking $\epsilon \rightarrow(1 / W)^{+}$. By [16] and Lemmas 6 and 7 .

$$
\log \mathcal{S}_{\mathbb{T}}^{*}\left((1 / W)^{+}\right):=\lim _{\epsilon \rightarrow(1 / W)^{+}} \log \mathcal{S}_{\mathbb{T}}^{*}(\epsilon)=\frac{1}{W} \log \mathcal{L}_{\mathbb{T}}^{*}\left(W^{-}\right)=\frac{\beta_{\mathbb{T}}^{0}}{W},
$$

so that $\mathcal{F}_{\mathbb{T}}^{*}(f) \sim f / W+\beta_{\mathbb{T}}^{0} / W$ as $f \rightarrow-\infty$. Recalling that $\mathcal{F}_{\mathbb{T}}(f)=\mathcal{F}_{\mathbb{T}}^{*}(f)$ completes the theorem.

Note that the arguments above also establish that

$$
\lim _{s \rightarrow \infty} \frac{1}{W s} \log \left(\sum_{n=W s}^{W s+c} p_{\mathbb{T}, n}^{*}(s+t)\right)=\lim _{s \rightarrow \infty} \frac{1}{n_{s}^{\max }} \log p_{\mathbb{T}, n_{s}^{\max }}^{*}(s)=\frac{\beta_{\mathbb{T}}^{0}}{W} .
$$

In particular, the polygons counted in the sum on the left include those which are maximally compressed, i.e. where $s=\lceil n / W\rceil$.

\section{Further results}

In addition to Theorem 1 , we are in fact able to determine the behaviour of $\mathcal{F}_{\mathbb{T}}(f)$ in the $f \rightarrow \infty$ limit.

Theorem 4 For any tube size $L \times M$, as $f \rightarrow \infty$ the free energy $\mathcal{F}_{\mathbb{T}}(f)$ is asymptotic to $f / 2$. That is,

$$
\lim _{f \rightarrow \infty}\left(\mathcal{F}_{\mathbb{T}}(f)-\frac{f}{2}\right)=0 .
$$

We also conjecture that the $f \rightarrow-\infty$ asymptote found in Theorem 1 is related to a certain subclass of $\mathcal{P}_{\mathbb{T}}$ polygons. We say a polygon $\pi$ of length $n$ and span $s$ is Hamiltonian if $\pi$ is also a full $(s+1)$-block; equivalently, the length and span satisfy $n=W(s+1)$. (Note that, because $n$ must be even, if $W$ is odd then such polygons only exist for odd $s$.) Let $\mathcal{P}_{\mathbb{T}}^{\mathrm{H}}$ be the set of such polygons, with $p_{\mathbb{T}, n}^{\mathrm{H}}$ being the number of length $n$. Eng (2014) has shown that such polygons have a growth rate, that is, the limit

$$
\kappa_{\mathbb{T}}^{\mathrm{H}}=\lim _{n \rightarrow \infty} \frac{1}{n} \log p_{\mathbb{T}, n}^{\mathrm{H}}
$$

exists. (One must take $n$ through multiples of $W$ if $W$ is even, or multiples of $2 W$ if $W$ is odd.)

Conjecture 1 The growth rates of full s-blocks (counted by span) and of Hamiltonian polygons (counted by length) are related by

$$
\kappa_{\mathbb{T}}^{\mathrm{H}}=\frac{\beta_{\mathbb{T}}^{0}}{W} .
$$

As a result, $\mathcal{F}_{\mathbb{T}}(f)$ is asymptotic to $\kappa_{\mathbb{T}}^{\mathrm{H}}+f / W$ as $f \rightarrow-\infty$.

The FWD-conjecture has been proved in Eng (2014) for Hamiltonian polygons. Hence, if Conjecture 1 is true, it will allow us to establish for $f \rightarrow-\infty$ that all but exponentially few sufficiently long polygons in a tube with $L \geq 2$ and $M \geq 1$ are knotted, extending the result that was previously known for any finite $f$. Conjecture 1 has been confirmed to hold for a number of small values of $L$ and $M$. A possible approach for establishing it for all tube sizes would be to find an appropriate upper bound on the polygon sum in 15 in terms of Hamiltonian polygons, but this is not straightforward. 


\section{References}

M. Atapour. Topological Entanglement Complexity of Systems of Polygons and Walks in Tubes. PhD thesis, University of Saskatchewan, 2008.

M. Atapour, C. E. Soteros, and S. G. Whittington. Stretched polygons in a lattice tube. Journal of Physics A: Mathematical and Theoretical, 42:322002, 2009.

R. Bauerschmidt, H. Duminil-Copin, J. Goodman, and G. Slade. Lectures on self-avoiding walks. In Probability and Statistical Physics in Two and More Dimensions, volume 15 of Clay Mathematics Proceedings, pages 395-467. American Mathematical Society, 2012.

N. R. Beaton. The critical pulling force for self-avoiding walks. Journal of Physics A: Mathematical and Theoretical, 48:16FT03, 2015.

N. R. Beaton, A. J. Guttmann, I. Jensen, and G. F. Lawler. Compressed self-avoiding walks, bridges and polygons. Journal of Physics A: Mathematical and Theoretical, 48:454001, 2015.

N. R. Beaton, C. E. Soteros, and J. W. Eng. Polygons in restricted geometries subjected to infinite forces. Submitted, preprint at arXiv:1604.07465, 2016.

J. Eng. Self-avoiding polygons in $(L, M)$-tubes. Master's thesis, University of Saskatchewan, 2014.

O. Farago, Y. Kantor, and M. Kardar. Pulling knotted polymers. Europhysics Letters, 60:53-59, 2002.

D. Ioffe and Y. Velenik. The statistical mechanics of stretched polymers. Brazilian Journal of Probability and Statistics, 24:279-299, 2010.

E. J. Janse van Rensburg. The Statistical Mechanics of Interacting Walks, Polygons, Animals, and Vesicles. Oxford University Press, 2000.

E. J. Janse van Rensburg, E. Orlandini, M. C. Tesi, and S. G. Whittington. Knotting in stretched polygons. Journal of Physics A: Mathematical and Theoretical, 41:015003, 2008.

J. Krawczyk, A. L. Owczarek, T. Prellberg, and A. Rechnitzer. Pulling absorbing and collapsing polymers from a surface. Journal of Statistical Mechanics - Theory and Experiment, page P05008, 2005.

E. Orlandini and S. G. Whittington. Statistical topology of closed curves: Some applications in polymer physics. Reviews of Modern Physics, 79:611-642, 2007.

N. Pippenger. Knots in random walks. Discrete Applied Mathematics, 25:273-278, 1989.

G. Rychlewski and S. G. Whittington. Self-avoiding walks and polymer adsorption: Low temperature behaviour. Journal of Statistical Physics, pages 1-8, 2011.

D. W. Sumners and S. G. Whittington. Knots in self-avoiding walks. Journal of Physics A: Mathematical and General, 21:1689, 1988.

C. Vanderzande. Lattice Models of Polymers. Cambridge University Press, 1998. 